\title{
A case of brain metastasis with pathological transformation of long-surviving malignant pleural mesothelioma: illustrative case
}

\author{
Ryuichi Noda, MD, ${ }^{1,2}$ Shunsuke Yanagisawa, MD, PhD, ${ }^{1,3}$ Masato Inoue, MD, PhD, ${ }^{1}$ and Tetsuo Hara, MD, PhD1 \\ ${ }^{1}$ Department of Neurosurgery, Center Hospital of the National Center for Global Health and Medicine, Shinjuku-ku, Tokyo, Japan; ${ }^{2}$ Department of Neurosurgery, NTT Medical \\ Center Tokyo, Shinagawa-ku, Tokyo, Japan; and ' ${ }^{2}$ epartment of Neurosurgery and Neuro-Oncology, National Cancer Center Hospital, Chuo-ku, Tokyo, Japan
}

BACKGROUND Malignant pleural mesothelioma (MPM) is a rare cancer, and in $80 \%$ of cases the cause is asbestos exposure. In 1972 , the World Health Organization (WHO) declared asbestos is a carcinogenic substance. Since then, every developed country has restricted and banned the product. Because of its high heat resistance, asbestos had been widely used as building material for decades. The WHO estimated that approximately 125 million people are exposed to asbestos, and more than 107,000 die from asbestos-related diseases annually. Because of its long incubation period, the number of patients is estimated to keep increasing in the near future.

OBSERVATIONS The authors report a case of long-surviving MPM with a rushed clinical course after brain metastasis. A 69-year-old woman diagnosed with MPM (epithelial type) 6 years earlier presented with a brain metastasis. The pathological result of the brain metastasis was the sarcomatoid type. This case showed the possibility of subtype transition after long survival.

LESSONS This article aids in understanding the long-term natural history of MPM and the possibility of epithelial-mesenchymal transition. Neurosurgeons have to be aware of its the natural history and the possibility of brain metastasis.

https://thejns.org/doi/abs/10.3171/CASE2099

KEYWORDS malignant pleural mesothelioma; asbestos; brain metastasis; epithelial-mesenchymal transition

Malignant pleural mesothelioma (MPM) is a rare cancer, and $80 \%$ of cases are caused by asbestos exposure. ${ }^{1}$ Because of its high heat resistance, asbestos had been widely used as building material for decades. The relation of asbestos to MPM was first reported in 1964. In 1972, the World Health Organization (WHO) and the International Agency for Research on Cancer declared asbestos is a carcinogenic substance. Since then, every Western country started to take measures by restricting or banning the use of asbestos. The WHO estimated approximately 125 million people worldwide are exposed to asbestos at their workplaces, and more than 107,000 workers die from asbestos-related diseases annually. According to Flanagan, the asbestos consumption in countries such as India, China, Russia, Brazil, and Indonesia totaled approximately one million metric tons in 2016. ${ }^{2}$ Chen et al. reported that China could face a public health crisis triggered by asbestos use in the near future because of its 30 - to 40 -year incubation period. ${ }^{3}$

Due to the lack of an established surgical treatment, not many patients match the operative indication. The treatment is multidisciplinary, including chemotherapy, radiation therapy, and surgery. Most of the patients are surgically untreatable, thus chemotherapy is the first-line treatment; however, the treatment is challenging and the prognosis is poor.

In 2007, pemetrexed (PEM) was approved for clinical use in Japan. Since then, PEM plus cisplatin (CDDP) combined therapy has been the first-line treatment, and long-surviving cases have been reported. We report a case of long-surviving MPM with its rushed clinical course after brain metastasis.

\section{Illustrative Case}

A 69-year-old-woman without any prior medical history, but with a history of asbestos exposure, was first diagnosed with MPM by partial resection of the pleura 6 years ago. The histological findings on hematoxylin-eosin staining of the resected tumor showed the epithelial type of MPM. Glandular, restiform, reticulated, or alveolar tumors grew invasively within the fibrous stroma of the pleura. It also had infiltration to the adipose tissue and striated muscle tissue in some areas. Papillary hyperplasia on the surface of the pleura was also observed.

ABBREVIATIONS EMT = epithelial-mesenchymal transition; $I M R T=$ intensity-modulated radiation therapy; $M P M=$ malignant pleural mesothelioma; $M R I=$ magnetic resonance imaging; $P E M=$ pemetrexed; $W H O=$ World Health Organization.

INCLUDE WHEN CITING Published January 18, 2021; DOI: 10.3171/CASE2099.

SUBMITTED November 3, 2020. ACCEPTED November 16, 2020.

(c) 2021 The authors, CC BY-NC-ND 4.0 (http://creativecommons.org/licenses/by-nc-nd/4.0/) 
TABLE 1. Results of immunohistochemical staining of the primary lesion and the brain lesion

\begin{tabular}{ccc}
\hline Staining & Primary Tumor & Brain Lesion \\
\hline Positive & AE1/AE3 & AE1/AE3 \\
\hline CAM5.2 & Calretinin \\
\hline Calretinin & D2-40 \\
\hline D2-40 & EMA \\
\hline HBME-1 & \\
\hline CD141 & \\
\hline EMA & WT-1 \\
\hline WT-1 & GFAP \\
\hline Desmin, CEA & \\
\hline TTF-1 & \\
\hline NapsinA &
\end{tabular}

Tumor cells showed a positive reaction for calretinin and D2-40 and a negative reaction for WT-1 on immunohistochemical staining (Table 1, Fig. 1A); p53 was negative and Ki67 was 10\% at most (Fig. 2).
The patient had undergone 6 courses of PEM+CDDP chemotherapy, followed by 60 courses of PEM as maintenance therapy. The MPM was a progressive disease when she switched to nivolumab 2 months prior to admission; she had just finished the 14th cycle. On the day of presentation, she was brought to our hospital by ambulance for a convulsive seizure. Head computed tomography showed a mass (2.5 cm in diameter) on the right parietal lobe. Because the tumor was located in the eloquent area and the size did not exceed $3 \mathrm{~cm}$, intensity-modulated radiation therapy (IMRT) (35 Gy/5 fr) was chosen as the initial treatment. One month after the treatment, tumor reduction was seen on gadolinium-enhanced magnetic resonance imaging (MRI) but showed reexpansion up to $3 \mathrm{~cm}$ in diameter the following month with neurological symptoms such as paralysis (Fig. 3A-C). Tumor resection was performed in order to improve the neurological symptoms. With the naked eye, we noted that the tumor was yellowish, elastic-hard, and well-circumscribed and measured $3 \times 2.5 \times$ $2.5 \mathrm{~cm}$ (Fig. 3D). We were able to perform a total resection of the tumor en bloc. On histological examination, dense proliferation of spindle-shaped tumor cells with a distorted enlarged nucleus formed loose bundles. Mitotic count was 20 in 10 high-power fields, with scattered coagulation and necrosis. There was no fibrosis in the tumor, and a region of sheet-like growth of round tumor cells with loose binding was seen. On immunohistochemical staining, a positive reaction was seen with AE1/AE3,

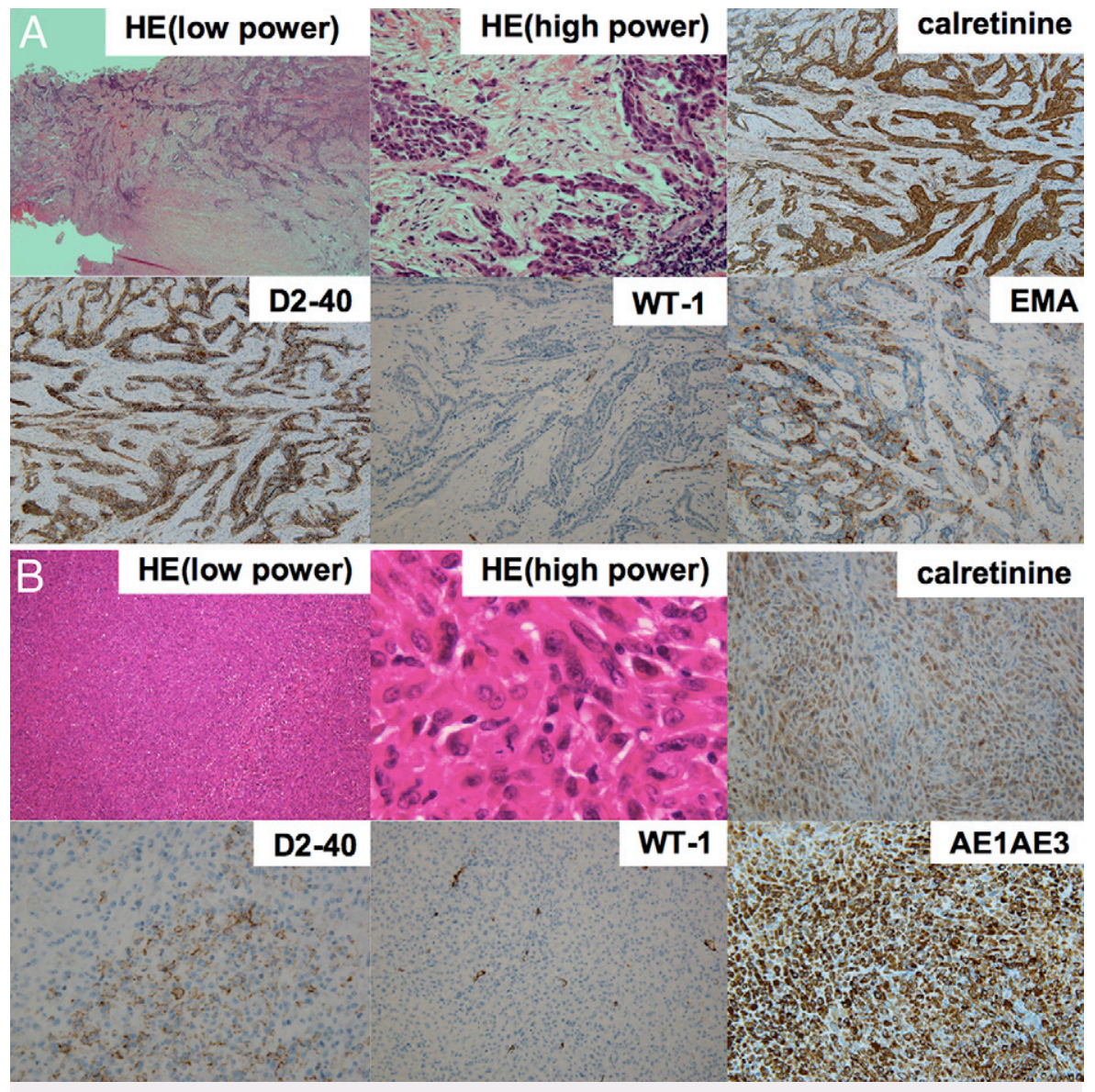

FIG. 1. A: Original magnifications $\times 20$ (HE[low power]), $\times 200$ (HE[high power]), and $\times 100$ (calretinine, D2-40, WT-1, and EMA). Histological results of the pleural lesion. The results revealed that the histological subtype was the epithelial type. B: Original magnifications $\times 20$ (HE[low power]), $\times 400$ (HE[high power]), and $\times 200$ (calretinine, D2-40, WT-1, and AE1AE3). Histological results of the brain lesion. The histological subtype was sarcomatoid. 

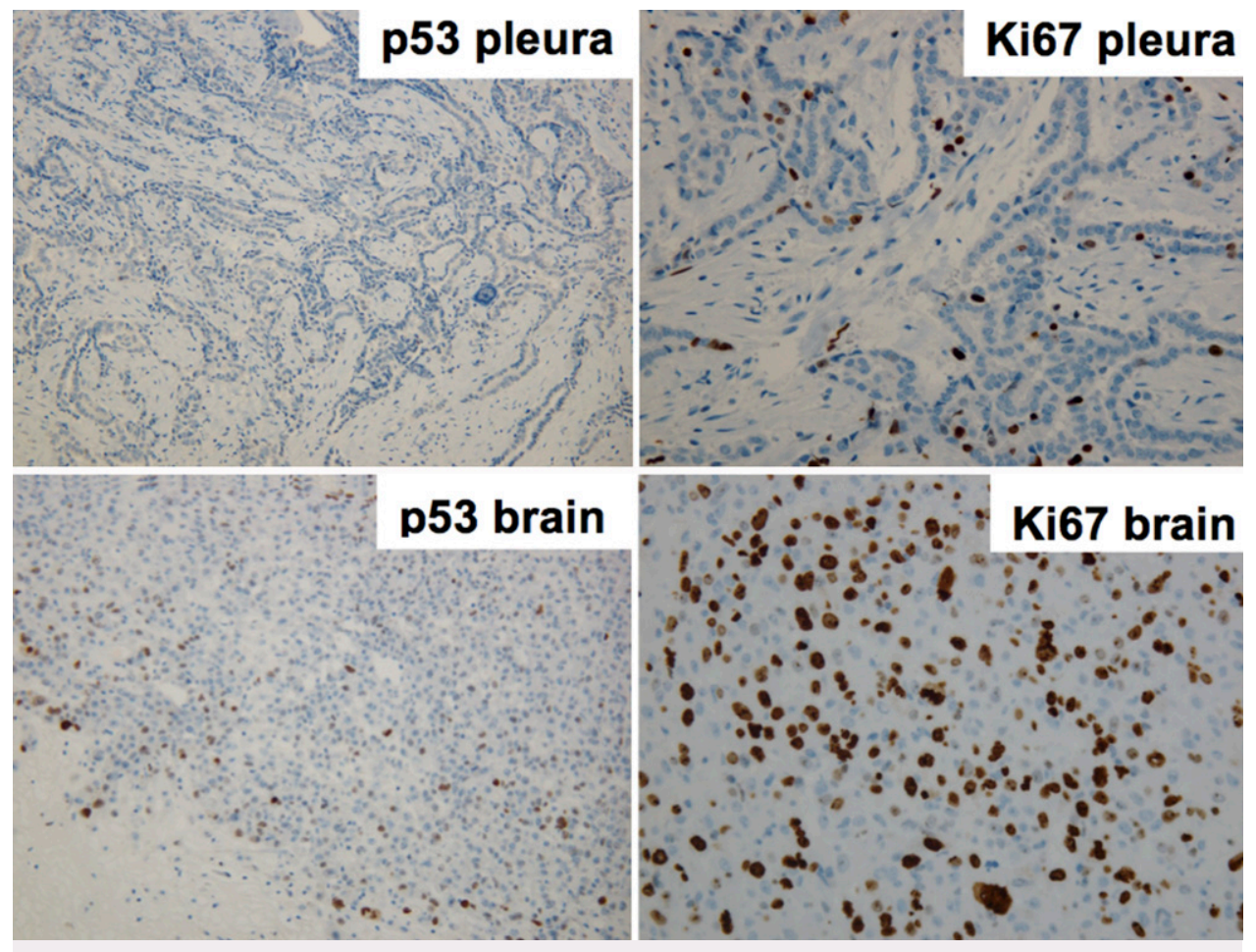

FIG. 2. Original magnifications $\times 200$ (left) and $\times 400$ (right). Results of immunohistochemical staining of $p 53$ and Ki67. Comparison between the pleural lesion and the brain metastasis. Both of the results were higher in the brain lesion.

calretinin, and D2-40; a negative reaction was seen with WT-1 and GFAP (Table 1, Fig. 1B); and p53 and Ki67 were $15 \%$ and $50 \%$, respectively (Fig. 2). Histological results were consistent with brain metastasis of MPM. However, the histopathological type was sarcomatoid MPM. Postoperative MRI showed no residual tumor. The patient was discharged from the hospital without symptoms. Two months and 4 months after the surgery, the patient had multiple increasing small brain metastases and received IMRT and IMRT with whole-brain radiation, respectively. The pleural tumor also showed rapid growth. The patient died 6 months after the operation.

\section{Discussion}

MPM are classified into 3 subtypes: epithelial, sarcomatoid, and biphasic; and these subtypes reflect the prognosis. MPM is thought to be a disease with a poor prognosis; the median survival time with each subtype is 15.2-16.9 months, 3.8-5.5 months, and 7.4-13.1 months, respectively., 4 Flores et al. reported that the 5 -year overall survival was $12 \%$; median survival was 38 months for stage I, 19 months for stage II, 11 months for stage III, and 7 months for stage IV patients. ${ }^{6}$ The median survival was 4-12 months without any treatment and 6-18 months with any treatment. ${ }^{7}$ All treatment results are not favorable, and there has been a long history of effort to find the cure, until the development of chemotherapy.

The possibility of long survival due to PEM is reported in few articles. ${ }^{4,8,9}$ Our case's clinical course before the introduction of nivolumab was similar to that of the previous cases. It is also notable that in our case the primary lesion was the epithelial type, which was a favorable prognosis factor. Both MIB-1 and p53 are also known to be poor prognostic factors. ${ }^{10,11}$ The MIB-1 index, p53 of the primary lesion, was negative, suggesting slow progression and relatively good prognosis.

Brain metastasis of MPM has been a rare condition due to the shortsurviving clinical course of MPM. It was first described in an autopsy report in 1973 by Grumme and Bingas; ${ }^{12}$ the first active management of cerebral metastasis of MPM was reported in 1993 by Wroński and Burt. ${ }^{13}$ The incidence is reported to be $2-3.8 \% .{ }^{14}$ The prognosis of MPM with brain metastasis is said to be poor. It is usually detected in the late stage of the disease, and survival after detection is on the order of
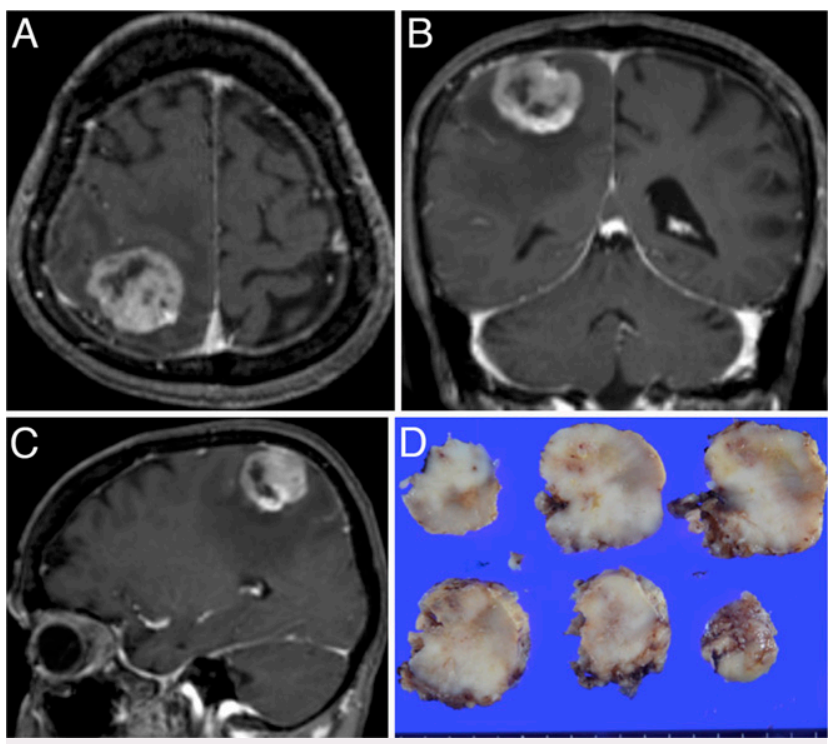

FIG. 3. Gadolinium-enhanced MRI (A, axial view; B, coronal view; C, sagittal view). Images of the removed tumor. The tumor was wellcircumscribed, avascular, with a homogeneously firm texture (D). 
weeks to months. ${ }^{14}$ Resection, whole-brain radiation, stereotactic radiation, systemic corticosteroids, intrathecal or systemic chemotherapy, and immunotherapy have been reported as treatment, but only a few cases have had a therapeutic response. ${ }^{14}$

\section{Observations}

In our case, the brain metastasis was found 7 years after the first diagnosis, and despite total resection, multiple recurrent metastases were seen on the postoperative MRI. A similar case was reported by Miller et al. ${ }^{14}$ In their report, a new larger remote cerebral lesion was identified 6 weeks after operation. In the literature, it is thought that the brain metastasis occurs after regression in response to systemic chemotherapy ${ }^{15}$ and recurs within 5 to 7 months after surgery. ${ }^{16}$ Our case had a treatment regression after 60 courses of PEM, which might have been the starting point of disease progression, including the brain metastasis.

Previous reports suggest that $11 \%$ of brain metastasis cases may differ histologically from the primary lesion as a worse subtype. ${ }^{14}$ Ota et al. formulated two hypotheses for this phenomenon. ${ }^{4}$ First, the primary lesion's histology was mostly epithelial but contained a small sarcomatoid component. Aggravation of the sarcomatoid component might have taken over the entire metastatic lesion during its long clinical course. Second, the epithelial MPM transformed into a malignant subtype due to the long-term use of chemotherapeutic medication. Fassina et al. referred to the possibility of epithelial-mesenchymal transition (EMT). ${ }^{17}$ EMT is defined as "the epithelial cell losing its nature, and acquiring the nature of mesenchymal cells, such as osteocytes, muscle cells, fibroblasts." 18 Kinoshita et al. also reported a case of histological transformation of the recurrent lesion after 14 years. ${ }^{19}$ In our case, the histological subtype between the primary and secondary lesion was proven to be different and EMT was suspected. We were able to acquire the MIB- 1 index, p53 of both the primary and brain lesions, and to compare the results. According to these results, there was a significant difference in the degree of malignancy between the two lesions. This result was compatible with previous reports. ${ }^{4}$ Our hypothesis is that the primary lesion transformed into a worse subtype and presented as a recurrent pleural lesion, progressed the disease, and finally translocated to the brain.

\section{Lessons}

Currently, brain metastasis of MPM is seldom seen and is still a rare condition. Because of the invention and development of chemotherapeutics, we suspect that the incidence of brain metastasis will increase due to the elongation of survival time. In conclusion, this article aids understanding of the long-term natural history of MPM, and neurosurgeons have to be aware of the natural history of MPM and the possibility of brain metastasis in long-term survivors.

\section{References}

1. Galetta D, Catino A, Misino A, etal. Sarcomatoid mesothelioma: future advances in diagnosis, biomolecular assessment, and therapeutic options in a poor-outcome disease. Tumori. 2016;102(2):127-130.

2. Flanagan DM. Asbestos (advance release). In: Minerals Yearbook. U.S. Geological Survey, U.S. Dept of the Interior; 2016.

3. Chen T, Sun XM, Wu L. High time for complete ban on asbestos use in developing countries. JAMA Oncol. 2019;5(6):779-780.

4. Ota S, Hara Y, Shinkai M, et al. An autopsy case of pleural mesothelioma that presented rapid progression and multiple organ metastasis after disease control for 5 years. Article in Japanese with English abstract. Ann Japanese Respir Soc. 2014;3:800-804.
5. Neumann V, Günthe S, Mülle KM, etal. Malignant mesotheliomaGerman mesothelioma register 1987-1999. Int Arch Occup Environ Health. 2001;74(6):383-395

6. Flores RM, Pass HI, Seshan VE, et al. Extrapleural pneumonectomy versus pleurectomy/decortication in the surgical management of malignant pleural mesothelioma: results in 663 patients. J Thorac Cardiovasc Surg. 2008;135(3):620-626.

7. Hughes RS. Malignant pleural mesothelioma. Am J Med Sci. 2005;329(1):29-44.

8. Kawamata O. A case of epithelial-type diffuse malignant pleural mesothelioma who survived with chemotherapy for 7 years. Article in Japanese with English abstract. Haigan. 2010;50:926-931.

9. Tanaka A, Takahashi T. Case of malignant pleural mesothelioma with long-term disease control after 4 different lines of systemic chemotherapy and pleurodesis. Article in Japanese. Nihon Kokyuki Gakkai Zasshi. 2009;47(5):383-387.

10. Comin CE, Anichini C, Boddi V, et al. MIB-1 proliferation index correlates with survival in pleural malignant mesothelioma. Histopathology. 2000;36(1):26-31.

11. Motta $A B$, Pinheiro $G$, Antonângelo L, et al. Morphological aspects as prognostic factors in malignant mesothelioma: a study of 58 cases. J Bras Pneumol. 2006;32(4):322-332.

12. Grumme T, Bingas B. Intracranial formation of metastases of diffuse pleuro-mesothelioma. Article in German. Zentralbl Neurochir. 1973;34(1):41-48.

13. Wroński M, Burt M. Cerebral metastases in pleural mesothelioma: case report and review of the literature. J Neurooncol. 1993;17(1): 21-26.

14. Miller AC, Miettinen M, Schrump DS, et al. Malignant mesothelioma and central nervous system metastases. Report of two cases, pooled analysis, and systematic review. Ann Am Thorac Soc. 2014;11(7):1075-1081.

15. Colleoni M, Liessi G, Avventi C, et al. Response to chemotherapy of brain metastases from malignant pleural mesothelioma. Tumori. 1996;82(5):456-458.

16. Ishikawa $\mathrm{T}$, Wanifuchi $\mathrm{H}, \mathrm{Abe} \mathrm{K}$, et al. Brain metastasis in malignant pleural mesothelioma presenting as intratumoral hemorrhage. Neurol Med Chir (Tokyo). 2010;50(11):1027-1030.

17. Fassina A, Cappellesso R, Guzzardo V, et al. Epithelial-mesenchymal transition in malignant mesothelioma. Mod Pathol. 2012;25(1): 86-99.

18. Kalluri R, Neilson EG. Epithelial-mesenchymal transition and its implications for fibrosis. J Clin Invest. 2003;112(12):1776-1784.

19. Kinoshita T, Ishii G, Matsumura $Y$, et al. The recurrence of malignant pleural mesothelioma 14 years after extrapleural pneumonectomy: possible histological transformation. Pathol Int. 2012;62(11):754-757.

\section{Disclosures}

The authors report no conflict of interest concerning the materials or methods used in this study or the findings specified in this paper.

\section{Author Contributions}

Conception and design: all authors. Acquisition of data: all authors. Analysis and interpretation of data: Yanagisawa, Hara. Drafting the article: Noda, Yanagisawa, Hara. Critically revising the article: Hara. Reviewed submitted version of manuscript: Hara. Approved the final version of the manuscript on behalf of all authors: Noda. Study supervision: Inoue, Hara.

\section{Correspondence}

Ryuichi Noda: NTT Medical Center, Tokyo, Japan. rnrn46_8447@ yahoo.co.jp. 